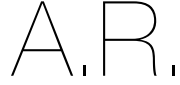

ARTIGO DE REVISÃO

${ }^{1}$ Escola Superior de

Tecnologias da Saúde de

Coimbra

Rua 5 de Outubro,

3046-854 Coimbra,

Portugal

"Endereço para correspondência:

Beatriz Mota Curado

Rua Maria Olguim,

$0.10,3^{\circ}$ direito

Tavarede, Figueira da Foz,

Portugal

beatriz.curado@hotmail.com

Histórico do artigo

Recebido a 26 de outubro de 2020 Aceite a 23 de maio de 2021

\section{INFLUÊNCIA DA SUPLEMENTAÇÃO DE SUMO DE BETERRABA NA MELHORIA DA PERFORMANCE EM ATLETAS DE CROSSFIT}

\author{
INFLUENCE OF BEETROOT JUICE SUPPLEMENTATION ON \\ PERFORMANCE IMPROVEMENT IN CROSSFIT ATHLETES
}

Beatriz Curado'"; Ana Faria'; Helena Loureiro'

RESUMO

INTRODUÇÃo: O CrossFit é uma prática desportiva predominantemente intermitente de alta intensidade e com uma forte componente de endurance. No sentido de melhorar a performance, o sumo de beterraba é um dos alimentos que tem sido amplamente utilizado como suplemento alimentar, no sentido de melhorar a performance. Várias investigações têm demonstrado melhorias na performance em modalidades que envolvam exercícios de endurance intensos e em exercícios de força e potência. Isto deve-se ao seu conteúdo em nitratos cuja evidência científica sugere apresentarem efeitos benéficos ao nível da vasodilatação, aumento do fluxo sanguíneo para as fibras musculares e promoção de trocas gasosas e regulação da eficiência da fosforilação oxidativa, para além de promoverem rápida recuperação muscular.

OBJETIVOS: O presente artigo de revisão tem por objetivos perceber quais são as variáveis da performance (força, potência ou endurance) influenciadas pelo sumo de beterraba, bem como as diferenças entre suplementação crónica ou aguda.

METODOLOGIA: Foi realizada pesquisa bibliográfica, nas bases de dados SCIELO e PUBMED, usando como critérios de inclusão: artigos publicados a partir de 2012 e redigidos em português ou inglês. Foram excluídos artigos cuja amostra era constituída por crianças ou idosos, realizados in vitro ou em animais, estudos em que não foram determinados os efeitos diretos do sumo de beterraba e em que não tenha sido reconhecida credibilidade académica.

RESULTADOS: A análise dos estudos revela a inconsistência de resultados em relação à influência do nitrato dietético nas várias componentes que influenciam a performance.

CONCLUSÕES: Sendo o CrossFit um exercício físico de minutos, no qual cada variável pode fazer a diferença, a utilização de nitratos em indivíduos responsivos, pode ser uma mais valia, apesar de não ter ficado comprovada a sua eficácia.

\section{PALAVRAS-CHAVE}

Sumo de beterraba, Nitratos, Performance, CrossFit, Treino intermitente de alta intensidade

\section{ABSTRACT}

INTRODUCTION: CrossFit is a predominantly intermittent sport of high intensity and with a strong endurance component. For this reason, beet juice is one of the foods that has been widespread as a supplement to improve performance. Several investigations had results in modalities that involve resistance exercises, strength and power exercises. This is due to its content in nitrates that have beneficial effects in vasodilation, increasing blood flow to the muscle fibers and promoting gas exchange and regulation of the efficiency of oxidative phosphorylation, in addition to promoting rapid muscle recovery.

OBJECTIVES: This review article aims to understand what are the performance variables (strength, power or endurance) influenced by the beet juice, as well as the differences between chronic or acute supplementation.

METHODOLOGY: Bibliographic research was carried out in the SCIELO and PUBMED databases, using as inclusion criteria: articles published since 2012 and written in Portuguese or English. Articles whose sample were children or elderly, performed in vitro or in animals, studies in which the direct effects of beet juice were not determined and in which academic credibility has not been recognized, were excluded

RESULTS: The analysis of the studies reveals the inconsistency of results regarding the influence of dietary nitrate on the various components that influence performance.

CONCLUSIONS: CrossFit is a physical exercise of minutes, in which each variable can make a difference, therefore the use of nitrates in responsive individuals can be an asset, although its effectiveness has not been proven.

KEYWORDS

Beetroot juice, Nitrates, Performance, CrossFit, High-intensity Intermittent training 


\section{INTRODUÇÃO}

O CrossFit é uma prática desportiva metabolicamente exigente cujo principal objetivo é maximizar a força e potência (1), para além de outros domínios da competência física como a resistência cardiorespiratória, flexibilidade, velocidade, coordenação, agilidade, equilíbrio e precisão $(2,3)$.

Esta metodologia de treino combina exercícios cardiovasculares, ginástica, movimentos com peso corporal e levantamento de peso (4). Inclui atividades anaeróbias e aeróbias, com diferentes combinações de exercícios, cargas e esquemas de repetição, sendo considerado um treino intervalado de alta intensidade (1-3).

Em todos os treinos existe um "treino do dia" ou WOD, que pode variar de 5 a 45 minutos e deverá ser realizado o mais rápido possíve (AFAP) ou concluído o máximo de trabalho num determinado período de tempo (AMRAP). A maior parte dos exercícios carecem de períodos de descanso prescritos, tornando o seu desempenho dependente da capacidade dos atletas sustentarem o trabalho em alta potência (1-3). Nos últimos anos, esta modalidade tem ganho popularidade por ser um desporto completo e por ser visível o seu impacto na composição corporal dos atletas. Também são notórios os efeitos psicológicos positivos, por ser uma modalidade desafiante, gera maior satisfação no cumprimento de metas, que por sua vez leva maiores níveis de adesão e motivação (3).

Os atletas, tal como a população geral, fazem escolhas alimentares tendo em consideração fatores fisiológicos, sociais, psicológicos e económicos. A melhoria da performance também poderá influenciar a escolha alimentar (5).

O sumo de beterraba (SB) concentrado é um dos alimentos que tem sido amplamente utilizado como suplemento alimentar em exercícios intermitentes de alta intensidade, como o CrossFit, no sentido de melhorar a performance.

Este sumo contém grande quantidade de nitrato inorgânico (NO3-), um percursor do óxido nítrico (NO) $(6,7)$, que promove a vasodilatação, aumentando o fluxo sanguíneo para as fibras musculares e promovendo trocas gasosas, a regulação da eficiência da fosforilação oxidativa e induz eficiência mitocondrial. Todos estes efeitos favorecem o metabolismo oxidativo (8-13).

Várias investigações têm demonstrado que a suplementação com SB poderá favorecer a performance em modalidades que envolvam exercícios de endurance intensos, nos quais o metabolismo energético é predominantemente oxidativo $(12,14,15)$, e também em exercícios de força e potência $(8,16,17)$.

\section{OBJETIVOS}

Tendo em conta os escassos, mas crescente número de trabalhos que investigaram os efeitos da suplementação com sumo de beterraba em exercícios intermitentes de alta intensidade, justifica-se a necessidade deste artigo de revisão para uniformizar resultados de estudos anteriores.

Assim sendo, o objetivo principal do presente artigo de revisão é perceber se o consumo de SB poderá melhorar a performance em atletas de CrossFit. Tendo como hipóteses $(H)$ :

H1: O consumo de SB aumentou a força e potência em exercícios intermitentes de alta intensidade.

H2: O consumo de SB aumentou a endurance em exercícios intermitentes de alta intensidade.

H3: A suplementação aguda com SB melhorou a performance de atletas em exercícios intermitentes de alta intensidade.

H4: A suplementação crónica com SB melhorou a performance de atletas em exercícios intermitentes de alta intensidade.

\section{METODOLOGIA}

Este estudo constitui uma revisão bibliográfica de caráter analítico a respeito da influência da suplementação de SB na melhoria da performance em atletas de CrossFit.

Após a escolha do tema, foi realizada a pesquisa bibliográfica, com recolha de dados no período de junho a agosto de 2020, tendo sido usadas as seguintes bases de dados: Scientific Eletronic Library Online (SCIELO) e National Library of Medicine (PUBMED).

A palavras-chave utilizada na pesquisa foram: beetroot juice ou beetroot ou nitrates ou nitric oxide e performance ou CrossFit ou high-intensity intermittent training.

O principal critério de seleção foi a relevância para a temática em estudo. Foi definido como critério de inclusão publicações a partir de 2012, pois foi observada escassez de artigos sobre esta temática no período anterior a 2010. No entanto, não foi utilizado o critério temporal para redação da introdução ou da discussão, podendo ser utilizados estudos realizados em qualquer época. Foram incluídos apenas artigos redigidos em português ou inglês. Devido à escassez de estudos cuja amostra fossem apenas atletas de CrossFit, foram incluídos, para além destes, atletas recreativos praticantes de treino de alta intensidade intermitente. Foram, ainda, aplicados critérios de exclusão para garantir a seleção de estudos desenhados com o objetivo único de determinar os efeitos da suplementação com sumo de beterraba em exercícios intermitentes de alta intensidade. Assim sendo, foram excluídos estudos com amostra constituída por crianças ou idosos (com $<18$ ou $>65$ anos), estudos realizados in vitro ou em animais e estudos em que não foram determinados os efeitos diretos do sumo de beterraba. Também foram excluídos artigos aos quais não tenha sido reconhecida credibilidade académica, ou seja, não referenciados com DOI, PMIB, ISBN ou ISSN. A Figura 1 representa o processo de seleção dos estudos incluídos no presente artigo de revisão. Após a seleção dos artigos conforme os critérios de inclusão previamente definidos, foram seguidos, nessa ordem, os seguintes passos: leitura exploratória; leitura seletiva e escolha do material que se adequam aos objetivos e tema deste estudo; leitura analítica e análise dos textos, finalizando com a realização de leitura interpretativa e redação.

\section{Figura 1}

Processo de seleção de artigos científicos

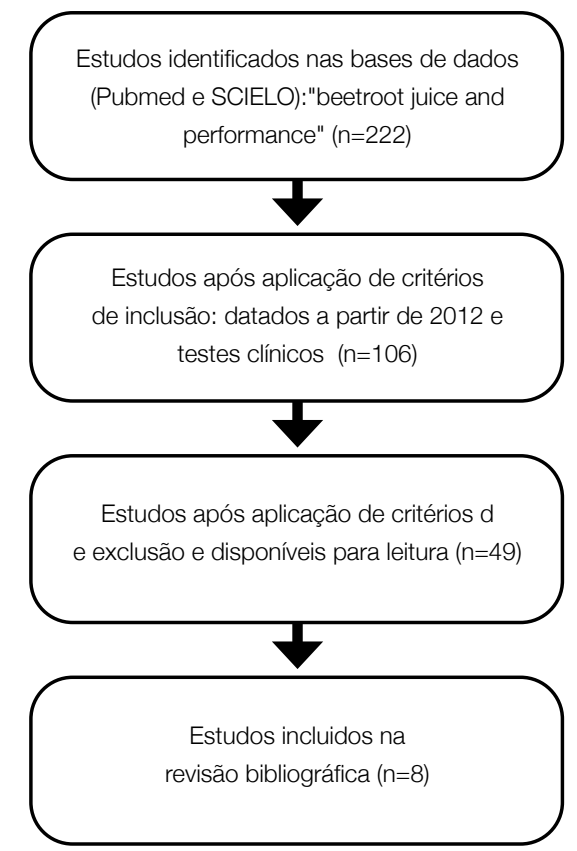




\section{RESULTADOS}

O presente artigo de revisão privilegiou estudos com amostra composta por atletas de CrossFit ou praticantes de treinos intermitentes de alta intensidade, e privilegiou estudos com utilização do teste Wingate, uma vez que, este avalia a capacidade de gerar potência explosiva de curta duração, uma caraterística fundamental em determinados exercícios de CrossFit.

Devido à natureza complexa deste tipo de treinos, os participantes seguem diferentes regimes e rotinas, daí a dificuldade, em alguns estudos, de quantificar e comparar componentes do regime de treino mais detalhadamente, tais como intensidade e volume, dando origem a resultados enviesados.

A análise dos estudos apresentados (Tabela 1) revela a inconsistência de resultados em relação à influência do nitrato dietético nas várias componentes que influenciam a performance.

\section{DISCUSSÃO DOS RESULTADOS}

Em exercícios intermitentes de alta intensidade, a suplementação com nitratos poderá traduzir-se em maior tolerância ao exercício e aumento do tempo até à exaustão, uma vez que a contração muscular é mais eficiente com menor custo energético total, permitindo poupar energia. Também se poderá traduzir na redução do consumo de oxigênio $\left(\mathrm{VO}_{2}\right)$ sem efeito na frequência cardíaca ou acumulação de lactato sanguíneo; na redução da pressão arterial em repouso, na melhoria da função cardiorrespiratória e do desempenho no limiar anaeróbio (9, 15, 18-23). A suplementação com SB também poderá estar associada a diminuição da fadiga muscular, pois ajuda a repor as reservas de fosfocreatina ou a evitar a depleção das mesmas. Em paralelo, poderá evitar o excesso de metabolitos como ADP e fosfatos inorgânicos que induzem fadiga muscular $(6,9,18)$.

Para além disto, o SB parece estar associado à contração muscular das fibras tipo II, com aumento da sua capacidade de produzirem força, o que pode ser vantajoso em esforços que envolvam estas fibras (6).

Poderá, ainda, atenuar o declínio na rapidez de tomada de decisão que normalmente ocorre durante exercícios intermitentes prolongados, sendo que este também é um fator determinante na performance (24). Ao nível da recuperação, a suplementação com nitratos também poderá ser uma vantagem, uma vez que têm capacidade de reduzir a dor após lesão e acelerar a recuperação do dano muscular. Por este motivo, parece ser uma estratégia utilizar SB no pós-treino ou entre bouts de exercício $(25,26)$. Para além disto, o SB também contém compostos antioxidantes com capacidade de inibir a produção de radicais livres e desta forma limitar o dano celular (27).

Ainda assim, no presente artigo de revisão, as hipóteses 1 e 2 anteriormente colocadas não foram comprovadas, uma vez que o sumo de beterraba não demonstrou aumentar a força, potência e capacidade de endurance em exercícios intermitentes de alta intensidade.

Assim sendo, os resultados do presente artigo de revisão, revelam-se contraditórios com estudos anteriores, uma vez que estes indicam que o nitrato dietético poderá melhorar a performance em exercícios intermitentes de alta intensidade e que deverá ser usado como estratégia nutricional (28).

\section{Tabela 1}

Resumo dos estudos sobre os efeitos do nitrato dietético na performance

\begin{tabular}{|c|c|c|c|c|}
\hline AUTOR & AMOSTRA & DESENHO DO ESTUDO & SUPLEMENTAÇÃO & CONCLUSÕES \\
\hline Kramer et al 2016 (31) & $\begin{array}{l}\text { Atletas de CrossFit } \\
(\mathrm{N}=12 ; \text { homens) }\end{array}$ & $\begin{array}{l}\text { Estudo randomizado, cruzado, } \\
\text { duplo-cego }\end{array}$ & $\begin{array}{l}8 \mathrm{mmol} \text { de nitrato de potássio } \\
\text { por dia, durante } 6 \text { dias }\end{array}$ & $\begin{array}{l}\text { - Aumento da potência pico no } \\
\text { teste Wingate } \\
\text { - Não houve melhoria na } \\
\text { performance em treino específico } \\
\text { de CrossFit }\end{array}$ \\
\hline Cuenca et al 2018 (32) & $\begin{array}{l}\text { Atletas com pelo menos } 18 \\
\text { meses de treino de resistência } \\
(\mathrm{N}=15 \text {; homens) }\end{array}$ & $\begin{array}{l}\text { Estudo randomizado, cruzado, } \\
\text { controlado por placebo, duplo- } \\
\text { cego }\end{array}$ & $\begin{array}{l}70 \mathrm{ml} \mathrm{SB}(6,4 \mathrm{mmol} \mathrm{NO} 3-) 3 \mathrm{~h} \\
\text { antes do teste físico }\end{array}$ & $\begin{array}{l}\text { - Melhorou a potência média, } \\
\text { a potência pico e o tempo até } \\
\text { atingir a potência pico no teste } \\
\text { Wingate } \\
\text { - Sem diferenças na fadiga entre } \\
\text { SB e placebo }\end{array}$ \\
\hline Jonvik et al 2020 (34) & $\begin{array}{l}\text { Atletas recreativos } \\
(\mathrm{N}=15 ; \text { homens })\end{array}$ & $\begin{array}{l}\text { Estudo randomizado, cruzado, } \\
\text { controlado por placebo, duplo- } \\
\text { cego }\end{array}$ & $\begin{array}{l}2 \times 70 \mathrm{ml} \mathrm{SB} \text { ( } 985 \mathrm{mg} \text { nitrato), } \\
\text { durante } 6 \text { dias }\end{array}$ & $\begin{array}{l}\text { - Aumento do nitrato sérico sem } \\
\text { melhorias na performance. } \\
\text { - Sem melhorias na força, } \\
\text { potência ou endurance muscular }\end{array}$ \\
\hline Kramer 2015 (33) & $\begin{array}{l}\text { Atletas de CrossFit } \\
(\mathrm{N}=12 ; \text { homens) }\end{array}$ & $\begin{array}{l}\text { Estudo randomizado, cruzado, } \\
\text { duplo-cego }\end{array}$ & $\begin{array}{l}8 \mathrm{mmol} \text { de nitrato de potássio } \\
\text { por dia, durante } 6 \text { dias }\end{array}$ & $\begin{array}{l}\text { - Não existiram melhorias na } \\
\text { performance em atletas de } \\
\text { CrossFit } \\
\text { - Não existiram melhorias na } \\
\text { força, potência ou endurance } \\
\text { dos atletas }\end{array}$ \\
\hline Collins and Kearns 2020 (36) & $\begin{array}{l}\text { Atletas recreativos (pelo menos } \\
3 \text { treinos semanais de alta } \\
\text { intensidade funcional) } \\
\text { ( } \mathrm{N}=24 \text {; homens e mulheres) }\end{array}$ & $\begin{array}{l}\text { Estudo randomizado, cruzado, } \\
\text { controlado por placebo }\end{array}$ & $\begin{array}{l}70 \mathrm{ml} \mathrm{SB}(4,2 \mathrm{mmol} \mathrm{NO} 3-) 2 \mathrm{~h} \\
\text { antes do teste }\end{array}$ & $\begin{array}{l}\text { - Sem efeitos na performance em } \\
\text { exercícios HIFT }\end{array}$ \\
\hline Aucouturier et al 2015 (20) & $\begin{array}{l}\text { Atletas recreativos em desportos } \\
\text { de equipa } \\
\text { ( } \mathrm{N}=12 \text {; homens) }\end{array}$ & $\begin{array}{l}\text { Estudo randomizado, cruzado, } \\
\text { único-cego }\end{array}$ & $\begin{array}{l}500 \mathrm{ml} \mathrm{SB}(680 \mathrm{mg} / \mathrm{l}) 2 \mathrm{~h} \text { antes do } \\
\text { teste e } 3 \text { dias antes }\end{array}$ & $\begin{array}{l}\text { - Aumento da tolerância } \\
\text { ao exercício intermitente } \\
\text { supramáximo } \\
\text { - Sem efeitos significativos no } \\
\text { aumento do } \mathrm{VO}_{2} \text { máx }\end{array}$ \\
\hline Clifford et al 2016 (26) & $\begin{array}{l}\text { Atletas recreativos em desportos } \\
\text { de equipa } \\
\text { ( } \mathrm{N}=20 \text {; homens) }\end{array}$ & $\begin{array}{l}\text { Estudo duplo-cego, controlado } \\
\text { por placebo, grupos } \\
\text { independentes }\end{array}$ & $\begin{array}{l}2 \times 250 \mathrm{ml} \mathrm{SB}(>143 \mathrm{mg} \text { nitrato } \\
\text { por garrafa) consumido no dia e } \\
\text { nos } 3 \text { dias seguintes }\end{array}$ & $\begin{array}{l}\text { - Aceleração da recuperação, } \\
\text { redução da dor após dano } \\
\text { muscular } \\
\text { - Sem influência na performance } \\
\text { em sprint }\end{array}$ \\
\hline Martin et al 2014 (37) & $\begin{array}{l}\text { Atletas recreativos em desportos } \\
\text { de equipa } \\
\text { ( } N=16 \text {; homens e mulheres) }\end{array}$ & $\begin{array}{l}\text { Estudo randomizado, cruzado, } \\
\text { duplo-cego }\end{array}$ & $\begin{array}{l}70 \mathrm{ml} \mathrm{SB}(0,3 \mathrm{~g} \text { de nitratos) } 2 \mathrm{~h} \\
\text { antes do exercício }\end{array}$ & $\begin{array}{l}\text { - Uma dose aguda de nitratos } \\
\text { reduziu a performance em treinos } \\
\text { intermitentes de alta intensidade }\end{array}$ \\
\hline
\end{tabular}


Também se alega que a suplementação com SB poderá melhorar a performance tanto ingerido de forma crónica ( 5 a $8 \mathrm{mmol} /$ dia durante 3 dias) (29) como de forma aguda (5 a 8 mmol 2,5 horas antes do exercício) $(22,30)$.

No entanto, a presente revisão bibliográfica não comprova as hipóteses 3 e 4 anteriormente apresentadas, porque não demonstrou que a suplementação com nitrato dietético, tanto de forma crónica como de forma aguda origine melhorias na performance em indivíduos praticantes de exercícios intermitentes de alta intensidade. Apesar de alguns dos estudos analisados revelarem melhorias no aumento da potência pico, reveladas pelo teste Wingate $(31,32)$, outros indicam que não existem melhorias na força, potência ou capacidade de endurance $(33,34)$.

Dois estudos $(31,32)$ que utilizaram quantidades e formas de administração de nitrato dietético diferentes $(6,4 \mathrm{mmol}$ em SB de forma aguda ou $8 \mathrm{mmol}$ em cápsulas durante 6 dias), não existiram diferenças nos resultados obtidos. Ambos revelaram melhorias da potência pico no teste Wingate, daí que não exista até ao momento um protocolo de administração de nitrato dietético consensual. A suplementação dietética com nitrato demonstrou ser inconclusiva em exercícios de alta intensidade e uma dose de $70 \mathrm{ml}$ de SB administrada de forma aguda tem um baixo impacto na performance $(36,37)$. Por outro lado, doses de $500 \mathrm{ml}$ de SB 3 dias antes do exercício (20) ou 3 dias após (26) parecem ter benefícios ao nível do aumento da tolerância ao exercício e aceleração da recuperação, respetivamente. Relativamente aos estudos que avaliaram a influência do sumo de beterraba na fadiga ou tolerância ao exercício, os resultados também foram dispares (20, 32).

Ainda assim, no que concerne às melhorias na recuperação do dano muscular ou lesões, os resultados dos estudos revelam-se favoráveis neste sentido (26). A principal limitação deste artigo de revisão é o reduzido número de artigos científicos existentes sobre o tema, principalmente por se tratar de um tipo de treino relativamente recente, o que revela a necessidade de serem realizados mais estudos, numa amostra alargada de atletas praticantes de CrossFit, de forma a avaliar a influência do nitrato dietético na performance.

\section{CONCLUSÕES}

Apesar de não existir consenso nos estudos analisados, nem no panorama científico global, acerca do benefício do SB na performance em treinos intermitentes de alta intensidade ou mais especificamente em treinos de CrossFit, na prática, a suplementação com nitrato dietético, poderá ser benéfica para indivíduos que respondem positivamente ao mesmo.

Sendo o CrossFit um método de treino predominantemente intermitente de alta intensidade e tendo uma forte componente de endurance, o consumo de SB poderá melhorar a performance em atletas que praticam a modalidade e favorecer a rápida recuperação muscular (35), apesar desta teoria ainda não ter sido comprovada. Em competição, especialmente em contexto de CrossFit que é um exercício físico de minutos e no qual cada variável pode fazer a diferença, os nitratos dietéticos poderão ser uma mais valia.

\section{REFERÊNCIAS BIBLIOGRÁFICAS}

1. Escobar KA, Morales J, Vandusseldorp TA. The Effect of a Moderately Low and High Carbohydrate Intake on Crossfit Performance. Int J Exerc Sci [Internet]. [cited 2019 Dec 1];9(3):460-70. Available from: http://www.ncbi.nlm.nih.gov/pubmed/27766133. 2. Claudino JG, Gabbett TJ, Bourgeois F, Souza H de S, Miranda RC, Mezêncio B, et al. CrossFit Overview: Systematic Review and Meta-analysis. Sports Medicine - Open. 2018. 3. Gianzina EA, Kassotaki OA. The benefits and risks of the high-intensity CrossFit training.
Sport Sciences for Health. 2019.

4. Maxwell C, Ruth K, Friesen C. Sports Nutrition Knowledge, Perceptions, Resources, and Advice Given by Certified CrossFit Trainers. Sports. 2017.

5. Birkenhead KL, Slater G. A Review of Factors Influencing Athletes' Food Choices. Sports Medicine. 2015.

6. Domínguez R, Maté-Muñoz JL, Cuenca E, García-Fernández P, Mata-Ordoñez F, Lozano-Estevan MC, et al. Effects of beetroot juice supplementation on intermittent high-intensity exercise efforts. Journal of the International Society of Sports Nutrition. 2018. 7. Jones AM. Influence of dietary nitrate on the physiological determinants of exercise performance: A critical review. Applied Physiology, Nutrition and Metabolism. 2014.

8. Lundberg JO, Weitzberg E, Gladwin MT. The nitrate-nitrite-nitric oxide pathway in physiology and therapeutics. Nature Reviews Drug Discovery. 2008.

9. Larsen FJ, Weitzberg E, Lundberg JO, Ekblom B. Effects of dietary nitrate on oxygen cost during exercise. Acta Physiol. 2007.

10. Ferguson SK, Hirai DM, Copp SW, Holdsworth CT, Allen JD, Jones AM, et al. Impact of dietary nitrate supplementation via beetroot juice on exercising muscle vascular control in rats. J Physiol. 2013.

11. Erzurum SC, Ghosh S, Janocha AJ, Xu W, Bauer S, Bryan NS, et al. Higher blood flow and circulating NO products offset high-altitude hypoxia among Tibetans. Proc Natl Acad Sci U S A. 2007.

12. Pinna M, Roberto S, Milia R, Marongiu E, Olla S, Loi A, et al. Effect of beetroot juice supplementation on aerobic response during swimming. Nutrients. 2014.

13. Vitale K, Getzin A. Nutrition and supplement update for the endurance athlete: Review and recommendations. Nutrients. 2019.

14. Puype J, Ramaekers M, Van Thienen R, Deldicque L, Hespel P. No effect of dietary nitrate supplementation on endurance training in hypoxia. Scand J Med Sci Sport. 2015. 15. Vanhatalo A, Bailey SJ, Blackwell JR, DiMenna FJ, Pavey TG, Wilkerson DP, et al. Acute and chronic effects of dietary nitrate supplementation on blood pressure and the physiological responses to moderate-intensity and incremental exercise. Am J Physiol - Regul Integr Comp Physiol. 2010.

16. Jones AM. Dietary nitrate supplementation and exercise performance. Sport Med. 2014. 17. Coggan AR, Peterson LR. Dietary Nitrate Enhances the Contractile Properties of Human Skeletal Muscle. Exerc Sport Sci Rev. 2018.

18. Bailey SJ, Winyard P, Vanhatalo A, Blackwell JR, DiMenna FJ, Wilkerson DP, et al. Dietary nitrate supplementation reduces the $\mathrm{O} 2$ cost of low-intensity exercise and enhances tolerance to high-intensity exercise in humans. J Appl Physiol. 2009.

19. Vanhatalo A, Fulford J, Bailey SJ, Blackwell JR, Winyard PG, Jones AM. Dietary nitrate reduces muscle metabolic perturbation and improves exercise tolerance in hypoxia. J Physiol. 2011.

20. Aucouturier J, Boissière J, Pawlak-Chaouch M, Cuvelier G, Gamelin FX. Effect of dietary nitrate supplementation on tolerance to supramaximal intensity intermittent exercise. Nitric Oxide - Biol Chem. 2015.

21. Mosher SL, Andy Sparks S, Williams EL, Bentley DJ, Naughton LRM. Ingestion of a nitric oxide enhancing supplement improves resistance exercise performance. J Strength Cond Res. 2016.

22. Lansley KE, Winyard PG, Bailey SJ, Vanhatalo A, Wilkerson DP, Blackwell JR, et al. Acute dietary nitrate supplementation improves cycling time trial performance. Med Sci Sports Exerc. 2011.

23. Larsen FJ, Weitzberg E, Lundberg JO, Ekblom B. Dietary nitrate reduces maximal oxygen consumption while maintaining work performance in maximal exercise. Free Radic Biol Med. 2010; 48 (2): 342-7.

24. Thompson C, Wylie LJ, Fulford J, Kelly J, Black MI, McDonagh STJ, et al. Dietary nitrate improves sprint performance and cognitive function during prolonged intermittent exercise. Eur J Appl Physiol. 2015.

25. Clifford T, Bell O, West DJ, Howatson G, Stevenson EJ. The effects of beetroot juice supplementation on indices of muscle damage following eccentric exercise. Eur J Appl Physiol. 2016.

26. Clifford T, Berntzen B, Davison GW, West DJ, Howatson G, Stevenson EJ. Effects of beetroot juice on recovery of muscle function and performance between bouts of repeated sprint exercise. Nutrients. 2016. 
27. Vulić JJ, Ćebović TN, Čanadanović VM, Ćetković GS, Djilas SM, Čanadanović-Brunet JM, et al. Antiradical, antimicrobial and cytotoxic activities of commercial beetroot pomace. Food Funct. 2013

28. Porcelli S, Pugliese L, Rejc E, Pavei G, Bonato M, Montorsi M, et al. Effects of a short-term high-nitrate diet on exercise performance. Nutrients. 2016.

29. Bailey SJ, Fulford J, Vanhatalo A, Winyard PG, Blackwell JR, DiMenna FJ, et al. Dietary nitrate supplementation enhances muscle contractile efficiency during knee-extensor exercise in humans. J Appl Physiol. 2010.

30. Rimer EG, Peterson LR, Coggan AR, Martin JC. Acute Dietary Nitrate Supplementation Increases Maximal Cycling Power in Athletes. Int J Sport Physiol Perform. 2015. 31. Kramer SJ, Baur DA, Spicer MT, Vukovich MD, Ormsbee MJ. The effect of six days of dietary nitrate supplementation on performance in trained CrossFit athletes. J Int Soc Sports Nutr. 2016; 13:39.

32. Cuenca E, Jodra P, Pérez-López A, González-Rodríguez LG, da Silva SF, Veiga-Herreros $\mathrm{P}$, et al. Effects of beetroot juice supplementation on performance and fatigue in a 30-s all-out sprint exercise: A randomized, double-blind cross-over study. Nutrients. 2018 33. Kramer SJ, Ormsbee MJ. The effects of six days of dietary nitrate supplementation on strength, power, and endurance in crossfit athletes. JissnBiomedcentralCom. 2015. 34. Jonvik KL, Hoogervorst D, Peelen HB, de Niet M, Verdijk LB, van Loon LJC, et al. The impact of beetroot juice supplementation on muscular endurance, maximal strength and countermovement jump performance. Eur J Sport Sci. 2020.

35. Frick S, Mayo J, Landry A, Harris M. The Effects of Beetroot Supplementation on Exercise Performance in Experienced CrossFit Athletes. J Acad Nutr Diet. 2018;118(10). 36. Collins SM, Kearns D. The effect of beetroot supplementation on high-intensity functional training performance. Int J Exerc Sci. 2020.

37. Martin K, Smee D, Thompson KG, Rattray B. No improvement of repeated-sprint performance with dietary nitrate. Int J Sports Physiol Perform. 2014. 